\title{
ORIGINAL ARTICLE Changes in thyroid hormone concentrations during neonatal extracorporeal membrane oxygenation
}

\author{
L Leeuwen ${ }^{1}$, AFJ van Heijst ${ }^{2}$, J van Rosmalen ${ }^{3}$, YB de Rijke ${ }^{4}$, LWJE Beurskens ${ }^{1}$, D Tibboel ${ }^{1}$, ELT van den Akker ${ }^{5}$ and H IJsselstijn ${ }^{1}$
}

\begin{abstract}
OBJECTIVE: Thyroid hormone concentrations can be disturbed during critical illness. Our aim was to determine changes in thyroid hormone concentrations during neonatal extracorporeal membrane oxygenation (ECMO).

STUDY DESIGN: We included 21 ECMO-treated neonates. Age-specific s.d. scores (SDS) of free and total thyroxine (FT4; TT4), reverse and total triiodothyronine ( $\mathrm{rT3}$; TT3), thyroid-stimulating hormone (TSH) and thyroxine-binding globulin (TBG) were determined at six fixed time-points. Data were analyzed using general linear models.

RESULTS: At baseline, mean SDS FT4 (-0.78, 95\% Cl: -1.37 to -0.19$)$, TT4 $(-1.97,95 \% \mathrm{Cl}:-2.76$ to -1.18$)$, TT3 $(-0.88,95 \% \mathrm{Cl}:-1.13$ to -0.63$)$, TSH $(-2.14,95 \% \mathrm{Cl}:-2.93$ to -1.35$)$ and TBG $(-3.52,95 \% \mathrm{Cl}:-4.55$ to -2.50$)$ were low with high mean SDS rT3 $(0.53,95 \%$ Cl: 0.28 to 0.78 ). One hour after start ECMO, $\Pi 44$, TSH and TBG had further declined; $12 \mathrm{~h}$ after start ECMO $\Pi 33$ had declined (all $P<0.05)$. After this decline, mean SDS TSH increased to the baseline level $12 \mathrm{~h}$ after start ECMO $(-2.50,95 \% \mathrm{Cl}:-3.22$ to -1.79$)$, and was higher than baseline $48 \mathrm{~h}$ after start ECMO $(-0.56,95 \% \mathrm{Cl}:-1.29$ to 0.17$)$. This TSH increase was followed by increases in TT4 and TT3. FT4 remained constant within the normal range during ECMO.

CONCLUSIONS: Thyroid hormone concentrations before ECMO were suggestive of non-thyroidal illness syndrome (NTIS). During ECMO, increases in TSH, TT4 and TT3 after an initial decline possibly reflect spontaneous restoration of the hypothalamic-pituitarythyroid axis. FT4 remained constant within the normal range. This suggests that thyroxine therapy is not required during ECMO.
\end{abstract}

Journal of Perinatology (2017) 37, 906-910; doi:10.1038/jp.2017.56; published online 27 April 2017

\section{INTRODUCTION}

Thyroid hormone concentrations can be affected by several factors including gestational age, ${ }^{1}$ cardiac surgery, ${ }^{2,3}$ use of drugs such as dopamine, ${ }^{4}$ and critical illness. ${ }^{5-7}$ Critically ill patients typically present with lower concentrations of total triiodothyronine (ТT3) and total thyroxine (TT4), increased reverse triiodothyronine (rT3) and low to normal thyroid-stimulating hormone (TSH) concentrations, which is known as non-thyroidal illness syndrome (NTIS) or euthyroid sick syndrome. ${ }^{5-7}$ The magnitude of changes in $\Pi 3$ and $\Pi 4$ concentrations has been related to the severity of illness. Both low $\Pi 33$ and low $\Pi 4$ have been associated with adverse patient outcomes such as increased mortality in neonatal, pediatric and adult patients. ${ }^{8-14}$

Recently we reported that $67.3 \%$ of neonates screened during extracorporeal membrane oxygenation (ECMO) had an aberrant screening result for congenital hypothyroidism. ${ }^{15}$ The one study so far on thyroid hormone concentrations during neonatal ECMO found a decline in all thyroid hormone concentrations in 14 critically ill neonates directly after the start of ECMO. ${ }^{16}$ It is not known, however, whether and how thyroid hormone concentrations change during and after neonatal ECMO, and whether there are any potential therapeutic consequences of changes. Therefore, we determined longitudinal changes in thyroid hormone concentrations during and after neonatal ECMO therapy.

\section{METHODS}

\section{Patients}

We included 21 neonates with a diagnosis of meconium aspiration syndrome (MAS) or congenital diaphragmatic hernia $(\mathrm{CDH})$, which are the two most common indications for neonatal ECMO treatment. ${ }^{17}$ Neonates were admitted to either of the two ECMO centers in the Netherlands (Erasmus MC-Sophia Children's Hospital, Rotterdam or Radboud University Medical Center-Amalia Children's Hospital, Nijmegen), both level III university hospitals. ECMO therapy was initiated in case of reversible severe respiratory failure with an estimated mortality risk of $>80 \%$ as described by Stolar et al. and on the standardized CDH-EURO consortium consensus treatment protocol. ${ }^{18,19}$

Relevant clinical data were collected including gestational age, birth weight, gender, age at start ECMO, duration of ECMO, neonatal screening results for congenital hypothyroidism and survival. Severity of illness was estimated with the Pediatric Risk of Mortality III (PRISM III) score and the Pediatric Logistic Organ Dysfunction-2 (PELOD-2) score. ${ }^{20,21}$ PRISM III is a physiology-based score for predicting the mortality risk in pediatric intensive care unit (PICU) patients. It is calculated from the most abnormal values of 17 physiologic variables obtained in the first $24 \mathrm{~h}$ of PICU care (score range: $0-74$ ). ${ }^{20}$ The PELOD-2 is a descriptive score that allows daily assessment of the severity of multiple organ dysfunction syndrome in PICU patients. It includes 10 variables involving five organ dysfunctions with a score range of $0-33 .{ }^{21}$ PELOD-2 scores were calculated for the $24 \mathrm{~h}$ prior to blood sampling. Use of dopamine $\leqslant 8 \mathrm{~h}$ prior to blood sampling was recorded because dopamine affects thyroid hormone concentrations within the first hours after initiation of treatment, and pre-treatment concentrations are reached within hours after discontinuation. ${ }^{22}$ None of the patients received supplemental levothyroxine at any time-point. At 2

${ }^{1}$ Department of Pediatric Surgery and Intensive Care, Erasmus MC-Sophia Children's Hospital, Rotterdam, The Netherlands; ${ }^{2}$ Department of Neonatology, Radboud University Medical Center-Amalia Children's Hospital, Nijmegen, The Netherlands; ${ }^{3}$ Department of Biostatistics, Erasmus MC, Rotterdam, The Netherlands; ${ }^{4}$ Department of Clinical Chemistry, Erasmus MC, Rotterdam, The Netherlands and ${ }^{5}$ Department of Pediatrics, Division of Pediatric Endocrinology, Erasmus MC-Sophia Children's Hospital, Rotterdam, The Netherlands. Correspondence: Dr H IJsselstijn, Department of Pediatric Surgery and Intensive Care, Erasmus MC-Sophia Children's Hospital, Room Number SK-1280, Wytemaweg 80, Rotterdam 3015 CN, The Netherlands.

E-mail: h.ijsselstijn@erasmusmc.nl

Received 6 December 2016; revised 22 March 2017; accepted 29 March 2017; published online 27 April 2017 
years of age, the Bailey Scales of Infant Development-Second EditionDutch version (BSID-II-NL) was used to assess cognitive outcomes of patients using the mental development index score. The mean score of the mental development index is 100 with a s.d. of $15 .^{23}$

Informed consent was obtained from parents. Ethical approval for this study was obtained from both local Ethics Committees (CMO 2005/253).

\section{ECMO procedure}

In the Erasmus MC-Sophia Children's Hospital, the ECMO circuit was primed with erythrocyte concentrate, Ringer's solution, albumin, Tris buffer, sodium bicarbonate, calcium gluconate and heparin. In the Radboud University Medical Center-Amalia Children's Hospital, the ECMO circuit was primed with erythrocyte concentrate, fresh frozen plasma (FFP), sodium bicarbonate and heparin. The total prime volume was approximately $350 \mathrm{ml}$ in both centers. Once the circuit was fully primed, ECMO cannulation was performed. The pediatric intensivist or neonatologist and pediatric surgeon decided on the choice of ECMO modality (veno-venous or veno-arterial).

\section{Thyroid function measurements}

Blood was taken from an arterial catheter at six fixed time-points: $1 \mathrm{~h}$ prior to ECMO (baseline value); $1,12,48$ and $72 \mathrm{~h}$ after start of ECMO; $>24 \mathrm{~h}$ after cessation of ECMO. Plain tubes were centrifuged and serum was stored at $-20^{\circ} \mathrm{C}$. Free thyroxine (FT4), $\Pi 44, \Pi T 3$ and TSH concentrations were determined using chemiluminescence assays (Vitros ECl; Ortho Clinical-Diagnostics, Rochester, NY, USA). rT3 was measured by radioimmunoassay (ZenTech S.A., Liége, Belgium). Thyroxine-binding globulin (TBG) was measured on an Immulite XPi system (Siemens Healthcare Diagnostics B.V., Los Angeles, (A, USA). The intra- and interassay coefficients of variation were $<5.4 \%$ for $\mathrm{FT} 4,<6.4 \%$ for $\Pi 4,<8.7 \%$ for $\mathrm{rT} 3,<4.3 \%$ for $T \mathrm{~T} 3,<4.1 \%$ for TSH and $<6.4 \%$ for TBG. Thyroid hormone concentrations and TBG were calculated into s.d. scores (SDS) using agespecific reference values. ${ }^{24}$ The normal range was considered as between -2 SDS and +2 SDS compared to the reference values, which are shown in Supplementary Table 1.

\section{Statistical analyses}

Baseline characteristics of MAS and CDH patients were compared using the Fisher's exact test for categorical variables. The independent samples $t$-test was used for normally distributed variables, and the Mann-Whitney U-test for continuous variables that were not normally distributed. Differences in thyroid hormone concentrations at baseline between survivors and nonsurvivors were compared using the Mann-Whitney U-test. We used general linear models to describe the longitudinal changes in thyroid hormone concentrations and TBG. The dependent variables in these general linear models were the SDS of FT4, TT4, rT3, TT3, TSH and TBG. The independent variables were gestational age, diagnosis, use of dopamine $\leqslant 8 \mathrm{~h}$ prior to the laboratory measurement, treatment center (use of FFP) and time-point (treated as a categorical variable). A random intercept was used in the general linear models to account for the within-subject correlations. Multicollinearity was assessed by calculating correlations between independent variables. The results of the general linear models are presented using the estimated marginal means, which are the predicted values of the dependent variable adjusted for covariates in the model, and the associated $95 \%$ confidence intervals (Cl). To estimate the effect of critical illness, PELOD-2 score and survival were added as independent variables in subsequent analyses. The statistical tests were two-sided and used a significance level of 0.05 . All analyses were performed using SPSS 21.0 for Windows (IBM Corporation, Armonk, NY, USA).

\section{RESULTS}

\section{Patients}

We included 21 ECMO-treated neonates diagnosed with either MAS $(n=11)$ or $\mathrm{CDH}(n=10)$. The $\mathrm{CDH}$ patients had a significantly lower gestational age than the MAS patients, and were all treated with veno-arterial ECMO (Table 1). The CDH patients had a significantly higher PRISM III score than the MAS patients. Mental development index scores of $\mathrm{CDH}$ and MAS patients were within the normal range of the Dutch norm population (within 1 SDS of
Table 1. Patient characteristics

\begin{tabular}{lccc}
\hline Characteristic & $\begin{array}{c}\text { MAS patients } \\
\mathrm{n}=11\end{array}$ & $\begin{array}{c}\mathrm{CDH} \text { patients } \\
\mathrm{n}=10\end{array}$ & P-value \\
\hline Gestational age (weeks) & $40.7 \pm 0.6$ & $37.6 \pm 1.6$ & $<0.001$ \\
Birth weight (grams) & $3.38 \pm 0.37$ & $3.00 \pm 0.62$ & 0.10 \\
Male & $6(54.5 \%)$ & $5(50.0 \%)$ & 1.00 \\
PRISM III score (first 24h & $13 \pm 6$ & $21 \pm 7$ & 0.01 \\
of PICU admission) & $4(36.4 \%)$ & $10(100 \%)$ & 0.004 \\
$\begin{array}{l}\text { Veno-arterial ECMO } \\
\text { Age start ECMO (days) }\end{array}$ & $3(1-4)$ & $1(1-2)$ & 0.31 \\
$\begin{array}{l}\text { Duration of ECMO } \\
\text { therapy (hours) }\end{array}$ & $146 \pm 80$ & $165 \pm 65$ & 0.56 \\
MDI $^{\mathrm{a}}$ & $92 \pm 8^{\mathrm{b}}$ & $93 \pm 16^{\mathrm{c}}$ & 0.97 \\
\hline
\end{tabular}

Abbreviations: $\mathrm{CDH}$, congenital diaphragmatic hernia; $\mathrm{ECMO}$, extracorporeal membrane oxygenation; MAS, meconium aspiration syndrome; MDI, mental development index; PICU, pediatric intensive care unit; PRISM III, Pediatric Risk of Mortality III. Data are expressed as mean \pm s.d., median

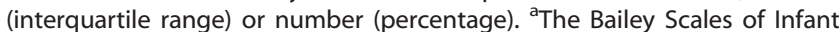
Development-Second Edition-Dutch version was used to assess mental development index in survivors. ${ }^{b}$ Nine MAS patients were tested: one patient died and one patient could not be tested because of severe mental disabilities due to perinatal asphyxia. 'Seven CDH patients were tested: two patients died and one patient was lost to follow-up.

Table 2. Thyroid hormone and thyroxine-binding globulin concentrations at baseline compared with the reference norms

\begin{tabular}{lcc}
\hline SDS of & ECMO patients $\mathrm{n}=21$ & P-value \\
\hline FT4 & $-0.78(-1.37$ to -0.19$)$ & 0.01 \\
TT4 & $-1.97(-2.76$ to -1.18$)$ & $<0.001$ \\
TT3 & $-0.88(-1.13$ to -0.63$)$ & $<0.001$ \\
rT3 & $0.53(0.28$ to 0.78$)$ & $<0.001$ \\
TSH & $-2.14(-2.93$ to -1.35$)$ & $<0.001$ \\
TBG & $-3.52(-4.55$ to -2.50$)$ & $<0.001$ \\
\hline
\end{tabular}

Abbreviations: $\mathrm{Cl}$, confidence interval; $\mathrm{ECMO}$, extracorporeal membrane oxygenation; FT4, free thyroxine; rT3, reverse triiodothyronine; SDS, s.d. score; TBG, thyroxine-binding globulin; TSH, thyroid-stimulating hormone; TT3, total triiodothyronine; TT4, total thyroxine. Data are presented using the estimated marginal means and the associated $95 \% \mathrm{Cls}$.

the mean). One MAS patient could not be tested because of severe mental disabilities due to perinatal asphyxia.

Eighteen patients were screened for congenital hypothyroidism in accordance with the Dutch neonatal screening protocol (others: screening too late $(n=2)$; screening result missing $(n=1))$. Two of the three (66.7\%) patients screened before ECMO had an aberrant initial screening result. Seven of the $12(58.3 \%)$ patients screened during ECMO had an aberrant initial screening result. None were diagnosed with congenital hypothyroidism. The three patients screened after ECMO all had a normal screening result. Three (14.3\%) patients died from pulmonary hypertension after ECMO decannulation: two $\mathrm{CDH}$ patients at the age of 9 and 11 days, respectively; one MAS patient at the age of 9 days.

Thyroid hormone and thyroxine-binding globulin concentrations at baseline

Mean baseline values of FT4, TT4, T3, TSH and TBG were significantly below the reference norm $(\mathrm{SDS}=0)$, and $\mathrm{rT3}$ was significantly higher than the norm (Table 2). Mean baseline values of TSH and TBG were abnormally low before ECMO. Baseline values did not differ between MAS patients and $\mathrm{CDH}$ patients or between patients in the two different treatment centers (data not 

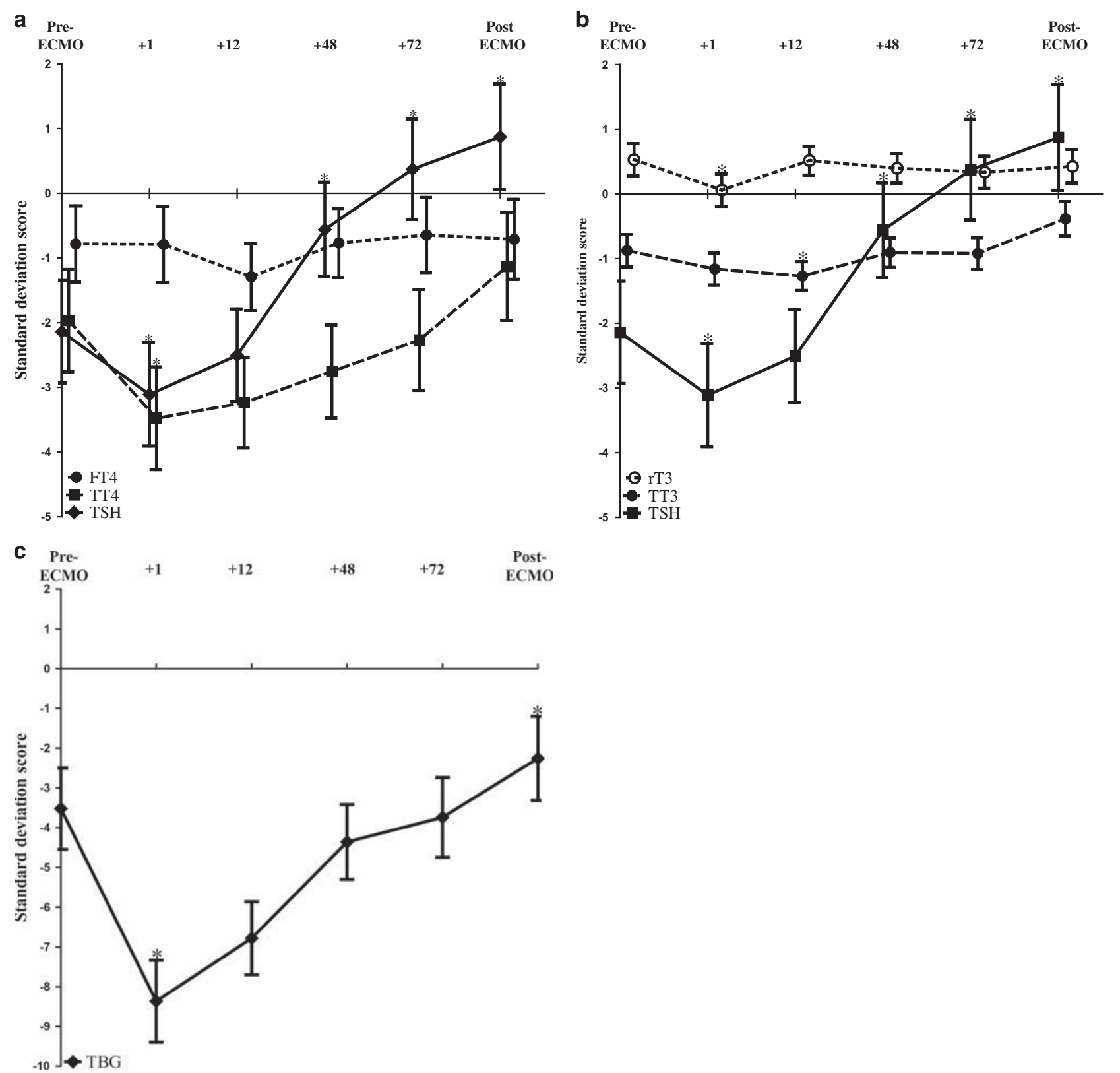

Figure 1. Changes in thyroid hormone and thyroxine-binding globulin concentrations during neonatal ECMO. (a) Changes in FT4, TT4 and TSH concentrations. (b) Changes in rT3, TT3 and TSH concentrations. (c) Changes in TBG concentrations. Estimated marginal means with error bars representing the $95 \% \mathrm{Cls}$ were plotted. Pre-ECMO: $1 \mathrm{~h}$ prior to ECMO (baseline value). $+1: 1 \mathrm{~h}$ after start of ECMO. $+12: 12 \mathrm{~h}$ after start of ECMO. +48: $48 \mathrm{~h}$ after start of ECMO. $+72: 72 \mathrm{~h}$ after start of ECMO. Post-ECMO: $>24 \mathrm{~h}$ after cessation of ECMO. * Indicates a significant change compared with the baseline value. Cl, confidence intervals; ECMO, extracorporeal membrane oxygenation; FT4, free thyroxine; rT3, reverse triiodothyronine; TBG, thyroxine-binding globulin; TSH, thyroid-stimulating hormone; TT3, total triiodothyronine; TT4, total thyroxine.

shown). At baseline, the three non-survivors had lower median TT3 SDS (-1.32, IQR: -1.46 to -1.29 vs -0.85 , IQR: -1.10 to -0.63$)$ and lower median TBG SDS ( -5.70 , IQR: -6.17 to -4.90 vs -2.71 , IQR: -3.89 to -1.69 ) than survivors (both $P=0.03$ ).

Thyroid hormone and thyroxine-binding globulin concentrations during neonatal ECMO

Figure 1 shows the changes in thyroid hormone concentrations. One hour after start of ECMO, all thyroid hormone concentrations and TBG showed a downward slope. Mean TT4, rT3, TSH and TBG significantly declined $1 \mathrm{~h}$ after start of ECMO $(P=0.003, P=0.002$, $P=0.02, P<0.001$, respectively). Mean $\Pi 3$ was significantly lower than baseline $12 \mathrm{~h}$ after start of ECMO $(P=0.02)$. After this initial decline, first TSH increased to baseline $12 \mathrm{~h}$ after start of ECMO. $\Pi 4, \mathrm{rT3}, \Pi 3$ and TBG reached their baseline values $48 \mathrm{~h}$ after start of ECMO. After the cessation of ECMO, TT3 and TSH concentrations were significantly higher than baseline $(P=0.009, P<0.001$, respectively). Mean TBG was still abnormally low after cessation of ECMO. FT4 concentrations did not significantly change during $\operatorname{ECMO}(P=0.53)$, and remained within the normal range.

Clinical variables associated with thyroid hormone and thyroxinebinding concentrations

The estimated coefficients of time point, gestational age, diagnosis of $\mathrm{CDH}$, dopamine use and treatment center (use of FFP) on thyroid hormone concentrations are given in Supplementary Table 2. The 
numbers of children that used dopamine are shown in Supplementary Table 3. Time point had a significant effect on all thyroid hormone and TBG concentrations, except FT4. Use of FFP was positively associated with TSH and TBG, but was negatively associated with FT4. A diagnosis of $\mathrm{CDH}$ was negatively associated with TSH. PELOD-2 score or survival had no significant associations with thyroid hormone concentrations or TBG (data not shown). Assessment of multicollinearity revealed a strong correlation between gestational age and diagnosis $(r=0.85)$. Excluding diagnosis as a covariate in the general linear models did not considerably change the estimated associations (data not shown).

\section{DISCUSSION}

We found that the concentrations of FT4, TT4, TT3, TSH and TBG were already low and the $\mathrm{rT3}$ concentration already high in critically ill neonates prior to the start of ECMO, resembling the pattern described in NTIS. ${ }^{5-7}$ The biologically active hormone FT4 was statistically significantly below the reference norm, but it remained within the normal range. After an initial decline directly after start ECMO, first TSH increased to a value above baseline $48 \mathrm{~h}$ after ECMO. This TSH increase was followed by increases in TT4 and $\Pi \pi 3$, possibly reflecting clinical recovery with restoration of the hypothalamic-pituitary-thyroid axis.

Stewart et al. studied thyroid hormone concentrations during ECMO in 14 neonates with severe respiratory failure from different causes, and also found a decline in all thyroid hormone concentrations directly after the start of ECMO. ${ }^{16}$ Further, a recent study in pediatric patients undergoing cardiac surgery with cardiopulmonary bypass specifically addressed the thyroid response in a subgroup of 57 neonates. ${ }^{3}$ Similar to our results, TSH strongly declined after surgery. However, they also found a small drop in FT4. Agus et al. ${ }^{25}$ found that low concentrations of thyroid hormones are present in ECMO prime fluid. In the current study, the prime volume was $350 \mathrm{ml}$. We think that the immediate decline of thyroid hormone concentrations can be ascribed to a dilutional effect. Based on the average birth weights in our study, we estimated that the dilutional factor in MAS was 55\% (350/(350 $+287) \times 100 \%)$ and in $\mathrm{CDH}$ was $58 \%(350 /(350+255) \times 100 \%)$. We think that this small difference in dilutional factor did not cause a significant difference in the decline of thyroid hormone levels between both groups. However, a larger difference in dilutional factor could lead to a different response. Agus et al. suggested that concentrations of thyroid hormones would be higher when FFP is used in the prime fluid. ${ }^{25}$ Indeed we found a positive association between TSH and TBG and the use of FFP. However, this positive association was not found for $\Pi 44, \mathrm{rT3}$ and $\Pi \mathrm{T} 3$, and contrary to this suggestion, we found that the use of FFPs was associated with lower FT4 levels. From this study, we cannot make a definite conclusion about the effect of the of FFP in prime fluid on thyroid hormone levels.

Hemodilution may not be the only explanation for this decline in thyroid hormone concentrations. The severe decline in TBG concentration could also be explained by a global inflammatory response with consequent capillary leakage, which occurs during the initial phases of cardiopulmonary bypass and ECMO. ${ }^{26-28}$ This capillary leak allows plasma proteins to move from the intravascular to the extravascular space, which lowers plasma protein concentrations.

Other factors contributing to the changes in thyroid hormone concentrations may be the severity of critical illness and use of dopamine. Previous studies in neonates have found a relation between low $\Pi 33$ and low $\Pi 44$ and adverse patient outcomes such as increased mortality, longer duration of mechanical ventilation and longer hospital stay. ${ }^{12-14}$ Therefore, we expected thyroid hormone concentrations to be lower in neonates with a greater severity of illness. Indeed, we found lower concentrations of TT3 and TSH at baseline in non-survivors. However, we did not find an association between the PELOD-2 score or survival and thyroid hormone concentrations in the general linear model. This could be due to the relatively small sample size. Secondly, although the PELOD-2 score has been validated for PICU patients, ${ }^{21,29}$ the score is affected by treatment such as ventilator settings and use of vasoactive drugs. Therefore, it does not solely reflect the severity of illness. Possibly, the PELOD-2 score does not distinguish enough between treatment effects and severity illness in this specific group of ECMO-treated neonates. Furthermore, dopamine could have played a role, as it has been found to suppress TSH concentrations. $^{22}$ Dopamine could be discontinued in most patients during ECMO, and we think this indeed played a role in the TSH increase during ECMO. However, we did not find a significant association between dopamine and thyroid hormone concentrations, which may be due to the influence of other factors that potentially affect thyroid hormone concentrations during ECMO.

In the present study, the combination of low $\Pi 3, \pi \mathrm{T} 4$ and TSH concentrations prior to the start of ECMO indicates an aberrant feedback regulation of the hypothalamic-pituitary-thyroid axis, as has been previously described during NTIS. ${ }^{5-7}$ During ECMO, however, first the TSH concentration significantly increased, which was later followed by an increase in $\Pi 4$ and $\Pi 3$. Other studies have found the same pattern of TSH, TT4 and TT3 increases at the start of restoration of the hypothalamic-pituitary-thyroid axis. ${ }^{30,31}$ Therefore, we think these changes in thyroid hormone concentrations during ECMO suggest clinical recovery with restoration of the hypothalamic-pituitary-thyroid axis.

In a previous study we found a higher incidence of aberrant screening results for congenital hypothyroidism in neonates screened before $(73.1 \%)$ or during ECMO $(67.3 \%)$ than in neonates screened after (31.4\%) ECMO. ${ }^{15}$ In the present study, aberrant screening results were found only in neonates screened before or during ECMO. None of the children with an aberrant screening result had congenital hypothyroidism. Because thyroid hormone concentrations significantly changed during ECMO, we recommend to repeat thyroid function tests after ECMO. Thyroid function tests should be repeated before the age of 14 days, however, because thyroxine treatment started later than this age can lead to intellectual disabilities. ${ }^{32}$

Treatment of low thyroid hormone concentrations during NTIS remains a matter of debate. As thyroid hormone is important for neurodevelopment during early life, a low concentration during this period could possibly affect neurodevelopmental outcomes. Yet, studies on thyroid supplementation therapy in infants with hypothyroxinemia of prematurity found no beneficial effect. ${ }^{33}$ Further, a recent study found that transient hypothyroxinemia of prematurity was not associated with adverse neurodevelopmental outcomes at the age of 19 years. ${ }^{34} \mathrm{~A}$ randomized controlled trial in pediatric patients undergoing cardiac surgery found that T3 supplementation in patients $<5$ months of age was associated with reduced time of mechanical ventilation. ${ }^{35}$ However, T3 supplementation in pediatric patients undergoing cardiopulmonary bypass has not been found to improve their long-term neurodevelopmental outcomes. ${ }^{36}$ We found a constant FT4 concentration during ECMO, which was only slightly lower than the reference norm. Further, cognitive outcomes of MAS and CDH patients were similar to cognitive outcomes of the norm population, except for one MAS patient with severe perinatal asphyxia. This, in combination with the lack of evidence for thyroid replacement therapy and the increases in thyroid hormone concentrations during ECMO suggest that early treatment is not required during ECMO. However, larger studies with long-term follow-up of neurodevelopmental outcomes should be performed to draw definite conclusions.

A strength of this study is the determination of all thyroid hormone concentrations and TBG at fixed time-points before, during and after neonatal ECMO. Furthermore, it is the first study 
that calculated these concentrations into SDS using recently established age-specific reference values. ${ }^{24} \mathrm{~A}$ limitation of this study is that we did not measure thyroid hormone concentrations in the prime fluid used during ECMO. Thyroid hormone concentrations in prime fluid could have varied. Measurements of thyroid hormone concentrations in the prime fluid would have given more information about the hemodilution effect during ECMO.

In conclusion, we found that thyroid hormone disturbances similar to NTIS were present in critically ill neonates prior to the start of ECMO. After an initial decline directly after the start of ECMO, first TSH increased, followed by $\Pi 4$ and $\Pi 3$. This may reflect restoration of the hypothalamic-pituitary-thyroid axis during ECMO. FT4 remained constant within the normal range during the ECMO course. These results suggest that thyroid hormone replacement therapy is not required during ECMO.

\section{CONFLICT OF INTEREST}

The authors declare no conflict of interest

\section{ACKNOWLEDGEMENTS}

We acknowledge Sanne Vijfhuize, Maria de Ridder and Hans van Toor for their help in data collection. We thank Ko Hagoort for editorial advice.

\section{REFERENCES}

1 Thorpe-Beeston JG, Nicolaides KH, Felton CV, Butler J, McGregor AM. Maturation of the secretion of thyroid hormone and thyroid-stimulating hormone in the fetus. N Eng J Med 1991; 324: 532-536.

2 Mainwaring RD, Lamberti JJ, Billman GF, Nelson JC. Suppression of the pituitary thyroid axis after cardiopulmonary bypass in the neonate. Ann thorac Surg 1994; 58: $1078-1082$.

3 Cantinotti M, Lorenzoni V, Storti S, Moschetti R, Murzi B, Marotta M et al. Thyroid and brain natriuretic peptide response in children undergoing cardiac surgery for congenital heart disease- age-related variations and prognostic value. Circ J 2013; 77: 188-197.

4 Surks Ml, Sievert R. Drugs and thyroid function. N Eng J Med 1995; 333: 1688-1694.

5 Boelen A, Kwakkel J, Fliers E. Beyond low plasma T3: local thyroid hormone metabolism during inflammation and infection. Endocr Rev 2011; 32: 670-693.

6 Van den Berghe G. Non-thyroidal illness in the ICU: a syndrome with different faces. Thyroid 2014; 24: 1456-1465.

7 Fliers E, Bianco AC, Langouche L, Boelen A. Thyroid function in critically ill patients. Lancet Diabetes Endocrinol 2015; 3: 816-825.

8 Slag MF, Morley JE, Elson MK, Crowson TW, Nuttall FQ, Shafer RB. Hypothyroxinemia in critically ill patients as a predictor of high mortality. JAMA 1981; 245: 43-45.

9 Rothwell PM, Lawler PG. Prediction of outcome in intensive care patients using endocrine parameters. Crit Care Med 1995; 23: 78-83.

10 den Brinker M, Joosten KF, Visser TJ, Hop WC, de Rijke YB, Hazelzet JA et al. Euthyroid sick syndrome in meningococcal sepsis: the impact of peripheral thyroid hormone metabolism and binding proteins. J Clin Endocrinol Metab 2005; 90: $5613-5620$.

11 Bello G, Pennisi MA, Montini L, Silva S, Maviglia R, Cavallaro F et al. Nonthyroidal illness syndrome and prolonged mechanical ventilation in patients admitted to the ICU. Chest 2009; 135: 1448-1454.

12 Kurt A, Aygun AD, Sengul I, Sen Y, Citak Kurt AN, Ustundag B. Serum thyroid hormones levels are significantly decreased in septic neonates with poor outcome. J Endocrinol Invest 2011; 34: e92-e96.

13 Goldsmit GS, Valdes M, Herzovich V, Rodriguez S, Chaler E, Golombek SG et al. Evaluation and clinical application of changes in thyroid hormone and TSH levels in critically ill full-term newborns. J Perinat Med 2011; 39: 59-64.

14 Lim DJ, Herring MK, Leef KH, Getchell J, Bartoshesky LE, Paul DA. Hypothyroxinemia in mechanically ventilated term infants is associated with increased use of rescue therapies. Pediatrics 2005; 115: 406-410.
15 Leeuwen L, van Heijst AF, Vijfhuize S, Beurskens LW, Weijman G, Tibboel D et al. Nationwide evaluation of congenital hypothyroidism screening during neonatal extracorporeal membrane oxygenation. Neonatology 2016; 111: 93-99.

16 Stewart DL, Ssemakula N, MacMillan DR, Goldsmith LJ, Cook LN. Thyroid function in neonates with severe respiratory failure on extracorporeal membrane oxygenation. Perfusion 2001; 16: 469-475.

17 Paden ML, Rycus PT, Thiagarajan RR, Registry E. Update and outcomes in extracorporeal life support. Semin Perinatol 2014; 38: 65-70.

18 Stolar CJ, Snedecor SM, Bartlett RH. Extracorporeal membrane oxygenation and neonatal respiratory failure: experience from the extracorporeal life support organization. J Pediatr Surg 1991; 26: 563-571.

19 Reiss I, Schaible T, van den Hout L, Capolupo I, Allegaert K, van Heijst A et al. Standardized postnatal management of infants with congenital diaphragmatic hernia in Europe: the CDH EURO Consortium consensus. Neonatology 2010; 98 : 354-364.

20 Pollack MM, Patel KM, Ruttimann UE. PRISM III: an updated Pediatric Risk of Mortality score. Crit Care Med 1996; 24: 743-752.

21 Leteurtre S, Duhamel A, Salleron J, Grandbastien B, Lacroix J, Leclerc F et al. PELOD-2: an update of the PEdiatric logistic organ dysfunction score. Crit Care Med 2013; 41: 1761-1773.

22 Van den Berghe G, de Zegher F, Lauwers P. Dopamine suppresses pituitary function in infants and children. Crit Care Med 1994; 22: 1747-1753.

23 van der Meulen BF, van der Ruiter SAJ, Lutje Spelberg HC, Smrkovsky M. Bayley scales of infant development - Second Edition - Nederlandse versie (BSID-II-NL). Harcourt Test Publishers: Amsterdam, 2002.

24 Lem AJ, de Rijke YB, van Toor H, de Ridder MA, Visser TJ, Hokken-Koelega AC. Serum thyroid hormone levels in healthy children from birth to adulthood and in short children born small for gestational age. J Clin Endocrinol Metab 2012; 97: 3170-3178.

25 Agus MS, Jaksic T. Critically low hormone and catecholamine concentrations in the primed extracorporeal life support circuit. ASAIO J 2004; 50: 65-67.

26 Fortenberry JD, Bhardwaj V, Niemer P, Cornish JD, Wright JA, Bland L. Neutrophil and cytokine activation with neonatal extracorporeal membrane oxygenation. J Pediatr 1996; 128: 670-678.

27 Kozik DJ, Tweddell JS. Characterizing the inflammatory response to cardiopulmonary bypass in children. Ann Thorac Surg 2006; 81: S2347-S2354.

28 Seghaye MC, Grabitz RG, Duchateau J, Busse S, Dabritz S, Koch D et al. Inflammatory reaction and capillary leak syndrome related to cardiopulmonary bypass in neonates undergoing cardiac operations. J Thorac Cardiovasc Surg 1996; 112: 687-697.

29 Leteurtre S, Duhamel A, Deken V, Lacroix J, Leclerc F, Groupe Francophone de Reanimation et Urgences $P$. Daily estimation of the severity of organ dysfunctions in critically ill children by using the PELOD-2 score. Crit Care 2015; 19: 324 .

30 Murzi B, lervasi G, Masini S, Moschetti R, Vanini V, Zucchelli G et al. Thyroid hormones homeostasis in pediatric patients during and after cardiopulmonary bypass. Ann Thorac Surg 1995; 59: 481-485.

31 Hamblin PS, Dyer SA, Mohr VS, Le Grand BA, Lim CF, Tuxen DV et al. Relationship between thyrotropin and thyroxine changes during recovery from severe hypothyroxinemia of critical illness. J Clin Endocrinol Metab 1986; 62: 717-722.

32 Leger J, Olivieri A, Donaldson M, Torresani T, Krude H, van Vliet G et al. European Society for Paediatric Endocrinology consensus guidelines on screening, diagnosis, and management of congenital hypothyroidism. J Clin Endocrinol Metab 2014; 99: 363-384.

33 Osborn DA, Hunt RW. Postnatal thyroid hormones for preterm infants with transient hypothyroxinaemia. Cochrane Database Syst Rev 2007; 1: CD005945.

34 Hollanders JJ, Israels J, van der Pal SM, Verkerk PH, Rotteveel J, Finken MJ et al. No association between transient hypothyroxinaemia of prematurity and neurodevelopmental outcome in young adulthood. J Clin Endocrinol Metab 2015; 100: 4648-4653.

35 Portman MA, Slee A, Olson AK, Cohen G, Karl T, Tong E et al. Triiodothyronine Supplementation in Infants and Children Undergoing Cardiopulmonary Bypass (TRICC): a multicenter placebo-controlled randomized trial: age analysis. Circulation 2010; 122: S224-S233.

36 Mittnacht J, Choukair D, Kneppo C, Brunner R, Parzer P, Gorenflo M et al. Long-term neurodevelopmental outcome of children treated with tri-iodothyronine after cardiac surgery: follow-up of a Double-Blind, Randomized, PlaceboControlled Study. Horm Res Paediatr 2015; 84: 130-136.

Supplementary Information accompanies the paper on the Journal of Perinatology website (http://www.nature.com/jp) 